\title{
Can the European EHR Exchange Format Support Shared Decision Making and Citizen-Driven Health Science?
}

\author{
Stefano BONACINA ${ }^{\mathrm{a}, 1}$ Sabine $\mathrm{KOCH}^{\mathrm{a}}$, Isabel MENESES ${ }^{\mathrm{b}}$, Catherine CHRONAKI ${ }^{\mathrm{c}}$ \\ ${ }^{a}$ Health Informatics Centre, LIME, Karolinska Institutet, Stockholm, Sweden \\ ${ }^{\mathrm{b}}$ Shared Services for Ministry of Health, EPE., Lisbon, Portugal \\ ${ }^{\mathrm{c}}$ HL7 Europe Foundation, Brussels, Belgium
}

\begin{abstract}
The European Commission published in 2019 the Recommendation on the European Electronic Health Record exchange format (EHRxF) to support citizens and healthcare providers in securely accessing and sharing EHRs. The European EHRxF is expected to contribute to the digital transformation of health and care in the digital single market empowering citizens and building a healthier society. This paper presents areas of work that need to be resolved for the European EHRxF to advance shared decision-making for patients and citizen-centered science.
\end{abstract}

Keywords. Interoperability, shared decision making, citizen science, citizen empowerment, person centered care, digital Health Policy

\section{Introduction}

The European EHRxF recommendation [1] presents the list of principles and guidelines for the creation and adoption of specifications that support citizens and health care providers in securely accessing and sharing Electronic Health Records (EHRs). At the same time, the recommendation connects the European EHRxF to key European initiatives for the advancement of the digital health infrastructure across the Union [2].

The recommendation recognizes the right of citizens to access and securely share their health data across borders, and describes significant benefits including quality of care for citizens, health care costs reduction, significant time savings and modernization of health systems. Noting that more than two million Europeans sought health care in another member state [3], the European Council conclusions in 2017 emphasized the need of member states to make their EHR systems interoperable, enabling citizens to have better control of their data [4] and become active agents of their own health journey.

With wide adoption of the European EHRxF, the level of interoperability will improve and information will be processed in a consistent manner across health systems, respecting the General Data Protection Regulation and using secure electronic identification and authentication means (eIDAS) supported by the EU cybersecurity framework and building upon the eHealth Digital Services Infrastructure (eHDSI) and

\footnotetext{
1 Corresponding Author: Stefano Bonacina, Health Informatics Centre, Department of Learning, Informatics, Management and Ethics, Karolinska Institutet, Stockholm, Sweden; E-mail: stefano.bonacina@ki.se.
} 
forthcoming myHealth@EU programmes. With the European EHRxF advancing the quality of data, large health data repositories will enable the use of new technologies such as big data analytics and artificial intelligence to fuel new scientific discoveries, support shared decision making, and provide effective decision aids to patients.

The recommendation presents a set of principles, a set of common technical specifications and a process to further the elaboration of the European EHRxF [1]. The suggested mechanisms include frameworks for security and trust at the national level using the tools created in eHDSI and create incentives of the use of interoperable EHRs in national digital health networks. To develop the basis for a uniform interoperable datasharing format framework, the intergovernmental eHealth cooperation action " $\mathrm{X}$ eHealth" was established in September 2020 [5]. X-eHealth aims to foster the uptake of the European EHRxF on regional, national and European level by extending the already in place Patient Summary and ePrescription services. This will be done by defining functional and technical requirements for the newly added use cases considered of highest priority: Laboratory Results, Medical Imaging and Reports, Hospital Discharge Reports and patient summaries for Rare and Undiagnosed Diseases.

This paper presents initial results of an exploratory study that is part of X-eHealth, aiming to explore the degree to which and under what conditions these priority specifications for the European EHRxF can be used to support shared decision making and citizen science. Shared decision making is defined as "collaborative process of reaching a decision" [6] and refers to the decisions that patients make about alternative health treatments and procedures, guided by healthcare professionals [7]. Citizen Science is defined as "the general public engagement in scientific research activities when citizens actively contribute to science either with their intellectual effort or surrounding knowledge or with their tools and resources", by Socientize Consortium [8]. This definition relates closely to the use of the European EHRxF for citizen science. European Commission considers "Citizen science" a significant domain [9], and results from proposed initiatives about "state of Citizen Science in Europe" [10] and "COVID-19related mobile apps" [11] have been published as reports, by the Joint Research Centre (JRC), the European Commission's science and knowledge service. The next sections present our methodology, results, discussion and conclusions on the next steps to be taken so that the European EHRxF is fit for shared decision making and citizen science.

\section{Methodology}

Starting point for this exploratory study was document analysis of the European EHRxF recommendation document and its annex [1]. Preliminary literature search investigated whether shared decision making and citizen science were part of studies about Electronic Health Records and their summaries. Relevant papers about the topic were analyzed. Further, Health Level 7 (HL7) International work on accelerators [12], which aims at developing global health data interoperability adopting HL7 Fast Healthcare Interoperability Resources (FHIR) standard, was analyzed, and material from the FHIR connectathon in 2021 was reviewed to elicit trends in standardization. Three different literature searches in PubMed/Medline were done using the following search strings: 1 ("citizen science"[MeSH] OR "citizen science"[Text word]) AND "Electronic Health Records"[MeSH]; 2 - "Decision Making, Shared"[MeSH] AND "Electronic Health Records"[MeSH]; 3 - ("Patient Generated Health Data" [Text word] OR "Patient Generated Health Data"[MeSH]) AND “Electronic Health Record”[MeSH]. 


\section{Results}

Shared decision making and Citizen Science have been identified as key issues to be addressed in the European EHRxF. From the document analysis of the recommendation, its annex, and the guidelines about the patient summary [13], guidance on how to use the specification to facilitate shared decision making was absent. In fact, the Patient summary does not include any sections devoted to shared decision making, besides the aim to support patient empowerment. From the literature search about "shared decision making" and "Electronic Health Record", five items have been identified. Results show that "clinician notes shared with the patients" [14] and "pattern of decision making discourse" [15] have to be taken into account for developing an enriched European EHRxF. The literature search about "Citizen Science" and "Electronic Health Record" gave no results (zero item). A more targeted search using "Patient Generated Health Data" and "Electronic Health Record" as search terms resulted in 24 hits. Some studies [16,17] reported about the adaptation of standards for integration of data from wearables into EHRs, in particular HL7 Common Document Architecture (CDA). Other studies point out the limited capacity of EHRs for direct patient input [18], insufficient integration of mental health apps with EHRs [19], and EHRs being inferior at storing patient contextual data such as patients' needs, values, goals, and preferences relevant to their care [20]. This limited support for direct patient engagement was confirmed in the recent FHIR connectathon, in the Patient Empowerment track.

As for HL7 accelerators [12], the "Argonaut Project" aims at developing FHIRbased API and Core Data Services specifications to speed up the sharing of data. These APIs have been largely adopted by health systems in the US and inspired developments in Canada, Australia, and the Nordics. Results from that project can be considered in implementing the modelling of datasets about shared decision making and citizen science (https://argonautwiki.hl7.org/Main_Page). Then, "The CARIN Alliance" project aims at enhancing the ability for consumers and their authorized caregivers to get, use, and share their digital health information. However, it seems the project is focusing on the US context as the concept of "Consumer-Directed Exchange" defined by HIPAA legislation needs to be satisfied (https://www.hl7.org/carin/). Finally, the "Da Vinci project" is for defining a holistic view, including payers and providers to benefit patient-centred care, again focusing in the US (https:/www.hl7.org/about/davinci/). These developments can inform similar activities aiming at including public and private payers in technical solutions adopting the European EHRxF.

The recent online HL7 FHIR Connectathon attracted more than 600 people (FCAT26 - https://confluence.hl7.org/display/FHIR/2021-01+Connectathon+26) and was an opportunity to validate several scenarios and proof of concept applications related to shared decision making, many of which associated with accelerators. Among them, Patient Empowerment and International Patient Summary (IPS) where quite well attended. Patient empowerment concerned use cases where patients requested changes to their health record. IPS introduced the notion of IPS on demand. The track Findable Accessible Interoperable Reusable (FAIR) was most relevant to our study in the direction of supporting citizen science. The FAIR track and HL7 Fairness for FHIR project (FHIR4FAIR Implementation Guide) supported by the European funded FAIR4Health (www.fair4health.eu) project explored metadata for health data sets and developed links to the Bricks activity by the US National Institute of Health (https://brics.cit.nih.gov). That work would be most relevant in the generation of high-quality health data sets appropriately targeted for citizen science. 


\section{Discussion}

Our results show that targeted use cases, datasets and associated metadata are needed for the European EHRxF to reliably support and promote citizen science and shared decision making perspectives. At present, literature searches involving "Citizen Science" and "Electronic Health Record" (as terms from MeSH vocabulary) did not give results, and we used "Patient Generated Health Data" as a sub-group to achieve more targeted results. The fact that Electronic Health Record frequently refers to data vetted by health professionals may explain this outcome. As an exploratory study, concepts included in the definition of "Citizen Science" should be used to further refine search strings for more extensively reviewing the area, complementing the knowledge gained from the different projects and connectathons, to also survey the feasibility of European EHRxF not only for information exchange, but also for analysis of longitudinal data. For future research, we suggest the following next steps to improve the suitability of the European EHRxF for citizen empowerment and further use of data:

1. Conduct in-depth literature review to elicit more detailed requirements for the European EHRxF to better serve citizen

2. Verify these requirements in a series of dedicated multi-stakeholder workshops with engagement of the industry including the civil society, startup cycles, researchers, policy makers, and standards development organizations.

3. Inform, educate and support the community about European EHRxF specifications and their support decision aids, shared decision-making, and citizen science focusing on metadata used in high-quality data sets.

4. Improve and refine the European EHRxF specifications to advance interoperability, quality, usability of the health data.

These steps should be taken with the initiative from the scientific community reaching out to the relevant stakeholders and the accelerated digitalization makes it urgent.

This preliminary research especially focuses on prospective exchange formats and implementable artifacts such as HL7 FHIR resources. To overcome the limitation to the level of information and data models, and reach context-sensitive level, aspects and perspectives of multiple domain such as patients' personal, social, occupational and environmental context and individual perspectives will be addressed (step 2 in the above list), including knowledge and skills issues and eHealth literacy. For enabling knowledge-based decision support, multiple knowledge spaces, their formalization and mapping based on domain, as well as, specific ontologies will be considered (steps 3 and 4 in the above list). In addition, standards such as ISO 23903 "Health informatics Interoperability and integration reference architecture - Model and framework" and previous literature on the topic, for instance [21], will be carefully examined.

\section{Conclusions}

For the European EHRxF to be successfully deployed in the areas of shared decision making and citizen science, it has to be known and be recognized by stakeholders including the civil society, academia, policy makers, and the industry. Tools need to be made available that incorporate the format in relevant applications. Guidance on the process of integrating the European EHRxF in technical solutions need to be developed and validated. Semantic, language and accessibility aspects need to be elaborated on. Besides connecting to the start-up cycles, connection to research networks, and 
facilitating communities of practice can help advance adoption and quality of the information shared in the European EHRxF.

\section{References}

[1] European Commission. Recommendation on a European Electronic Health Record exchange format (C(2019)800) of 6 February 2019. [Internet, Updated 29 September 2020]. Available at URL: https://ec.europa.eu/newsroom/dae/document.cfm?doc id $=57253$

[2] European Commission. Communication on enabling the digital transformation of health and care in the Digital Single Market; empowering citizens and building a healthier society, April 25, 2018, https://ec.europa.eu/digital-single-market/en/news/communication-enabling-digital-transformationhealth-and-care-digital-single-market-empowering

[3] Directive 2011/24/EU of March 9, 2011 on the application of patients' rights in cross border healthcare (OJ L88, 4.4.11, p 45)

[4] Council of the European Union. Council conclusions on Health in the Digital Society - making progress in data-driven innovation in the field of health $(2017 / \mathrm{C} 440 / 05)$

[5] X-eHealth: eXchanging electronic Health Records in a common framework Support Action. https://cordis.europa.eu/project/id/951938

[6] MeSH. Decision Making, Shared. Retrieved 2020 at URL: https://www.ncbi.nlm.nih.gov/mesh/2030979

[7] King JS, Moulton BW. Rethinking informed consent: the case for shared medical decision-making. Am J Law Med. 2006;32(4):429-501.

[8] Socientize Consortium. Green paper on citizen science. Citizen Science for Europe. Towards a better society of empowered citizens and enhanced research. European Commission. Brussels. 2013, p. 6.

[9] European Commission. Citizen science. [Internet, updated 29 October 2020]. Available at URL: https://ec.europa.eu/digital-single-market/en/citizen-science

[10] Schade S, Tsinaraki C, Manzoni M, Berti Suman A, Spinelli FA, Mitton I, Kotsev A, Delipetrev B, Fullerton KT. Activity Report on Citizen Science 2020 - discoveries from a five year journey, EUR 30551 EN, Publications Office of the European Union, Luxembourg, 2020, ISBN 978-92-76-28370-6, doi: $10.2760 / 841551$, JRC123500

[11] Tsinaraki C, Mitton I, Dalla Benetta A, Micheli M, Kotsev A, Minghini M, Hernandez L, Spinelli F, Schade S. Analysing mobile apps that emerged to fight the COVID-19 crisis, European Commission, Ispra, 2020, JRC 123209.

[12] HL7 International. The HL7 FHIR Accelerator Program. [Internet 2021].Available at URL: https://www.hl7.org/about/fhir-accelerator/

[13] eHealth Network. Guideline on the electronic exchange of health data under Cross-Border Directive 2011/24/EU Release 2 Patient Summary for unscheduled care, 2016.

[14] Fossa AJ, Bell SK, DesRoches C. OpenNotes and shared decision making: a growing practice in clinical transparency and how it can support patient-centered care. JAMIA 2018;25(9):1153-59.

[15] Kruser JM, Benjamin BT, Gordon EJ, Michelson KN, Wunderink RG, Holl JL, Schwarze ML. Patient and Family Engagement During Treatment Decisions in an ICU: A Discourse Analysis of the Electronic Health Record. Crit Care Med. 2019;47(6):784-791.

[16] Plastiras P, O'Sullivan D. Exchanging personal health data with electronic health records: A standardized information model for patient generated health data and observations of daily living. Int J Med Inform. 2018;120:116-125.

[17] Maggi N, Douglas Magnoni L, Ruggiero C, Gazzarata R, Giacomini M. Information Technology System Including Patient Generated Health Data for Cancer Clinical Care and Research. Stud Health Technol Inform. 2019;261:289-293.

[18] Lavallee DC, Chenok KE, Love RM, Petersen C, Holve E, Segal CD, Franklin PD. Incorporating PatientReported Outcomes Into Health Care To Engage Patients And Enhance Care. Health Aff (Millwood). 2016;35(4):575-82.

[19] Wu DTY, Xin C, Bindhu S, Xu C, Sachdeva J, Brown JL, Jung H. Clinician Perspectives and Design Implications in Using Patient-Generated Health Data to Improve Mental Health Practices: Mixed Methods Study. JMIR Form Res. 2020;4(8):e18123.

[20] Holt JM, Cusatis R, Asan O, Williams J, Nukuna S, Flynn KE, Moore J, Crotty BH. Incorporating patientgenerated contextual data into care: Clinician perspectives using the Consolidated Framework for Implementation Science. Healthc (Amst). 2020;8(1):100369.

[21] Blobel B, Oemig F. The importance of architectures for interoperability. Stud Health Technol Inform. 2015;211:18-56. 